\title{
Stage IIA Pancreatic Cancer AJCC v6 and v7
}

National Cancer Institute

\section{Source}

National Cancer Institute. Stage IIA Pancreatic Cancer A/CC v6 and v7. NCI Thesaurus. Code C88102.

Stage IIA includes: T3, NO, M0. T3: Tumor extends beyond the pancreas but without involvement of the celiac axis or the superior mesenteric artery. N0: No regional lymph node metastasis. M0: No distant metastasis. (from AJCC 7th Ed.) 